\title{
Pinned distance problem, slicing measures and local smoothing estimates
}

\author{
Alex Iosevich and Bochen Liu
}

\begin{abstract}
We improve the Peres-Schlag result on pinned distances in sets of a given Hausdorff dimension. In particular, for Euclidean distances, with

$$
\Delta^{y}(E)=\{|x-y|: x \in E\},
$$

we prove that for any $E, F \subset \mathbb{R}^{d}$, there exists a probability measure $\mu_{F}$ on $F$ such that for $\mu_{F}$-a.e. $y \in F$,

- $\operatorname{dim}_{\mathcal{H}}\left(\Delta^{y}(E)\right) \geq \beta$ if $\operatorname{dim}_{\mathcal{H}}(E)+\frac{d-1}{d+1} \operatorname{dim}_{\mathcal{H}}(F)>d-1+\beta$

- $\Delta^{y}(E)$ has positive Lebesgue measure if $\operatorname{dim}_{\mathcal{H}}(E)+\frac{d-1}{d+1} \operatorname{dim}_{\mathcal{H}}(F)>d$;

- $\Delta^{y}(E)$ has non-empty interior if $\operatorname{dim}_{\mathcal{H}}(E)+\frac{d-1}{d+1} \operatorname{dim}_{\mathcal{H}}(F)>d+1$.

We also show that in the case when $\operatorname{dim}_{\mathcal{H}}(E)+\frac{d-1}{d+1} \operatorname{dim}_{\mathcal{H}}(F)>d$, for $\mu_{F}$-a.e. $y \in F$,

$$
\left\{t \in \mathbb{R}: \operatorname{dim}_{\mathcal{H}}(\{x \in E:|x-y|=t\}) \geq \operatorname{dim}_{\mathcal{H}}(E)+\frac{d+1}{d-1} \operatorname{dim}_{\mathcal{H}}(F)-d\right\}
$$

has positive Lebesgue measure. This describes dimensions of slicing subsets of $E$, sliced by spheres centered at $y$.

In our proof, local smoothing estimates of Fourier integral operators (FIO) plays a crucial role. In turn, we obtain results on sharpness of local smoothing estimates by constructing geometric counterexamples.
\end{abstract}

\section{Introduction}

1.1. Distance problem and pinned distance problem. One of the most important open problems in geometric measure theory is the Falconer distance conjecture ([4]). It says that given any set $E \subset \mathbb{R}^{d}, d \geq 2$, its distance set

$$
\Delta(E)=\{|x-y|: x, y \in E\}
$$

has positive Lebesgue measure whenever $\operatorname{dim}_{\mathcal{H}}(E)>\frac{d}{2}$. The best currently known results are due to Wolff in $\mathbb{R}^{2}([\mathbf{2 0}])$ and Erdogan in higher dimensions ([2]). They proved that $\Delta(E)$ has positive Lebesgue measure whenever $\operatorname{dim}_{\mathcal{H}}(E)>\frac{d}{2}+\frac{1}{3}$. There are also results where $E$ is assumed to have special structures $([6],[14],[16])$.

This work was partially supported by the NSA Grant H98230-15-1-0319. 
More generally, we may ask how large the Hausdorff dimensions of $E \subset \mathbb{R}^{d}$ need to be to ensure the set

$$
\Delta_{\Phi}(E)=\{\Phi(x, y): x, y \in E\}
$$

has positive Lebesgue for suitable functions $\Phi: \mathbb{R}^{d} \times \mathbb{R}^{d} \rightarrow \mathbb{R}$. In [3], Eswarathasan, Iosevich and Taylor prove that if $\Phi: \mathbb{R}^{d} \times \mathbb{R}^{d} \rightarrow \mathbb{R}$ has non-zero Monge-Ampere determinant (also called Phong-Stein rotational curvature condition), i.e.

$$
\operatorname{det}\left(\begin{array}{cc}
0 & \nabla_{x} \Phi \\
\nabla_{y} \Phi & \frac{\partial^{2} \Phi}{\partial x \partial y}
\end{array}\right) \neq 0,
$$

then $\Delta_{\Phi}(E)$ has positive Lebesgue measure whenever $\operatorname{dim}_{\mathcal{H}}(E)>\frac{d+1}{2}$.

A corollary of this result is that if $E$ is a closed subset of a closed compact Riemannian manifold $M$ of dimension $d \geq 2$ of Hausdorff dimension $>\frac{d+1}{2}$, then $\Delta_{\rho}(E)$ has positive Lebesgue measure, where $\rho$ denotes the Riemannian metric on $M$.

Another interesting version of the Falconer distance problem is the pinned distance problem. Given $E \subset \mathbb{R}^{d}$, we ask whether for "many" points $y \in \mathbb{R}^{d}$, the pinned distance set

$$
\Delta^{y}(E)=\{|x-y|: x \in E\}
$$

has positive Lebesgue measure. This problem was first studied by Peres and Schlag ([15]). They considered a very large class of functions $\Phi(x, y)$ that generalize orthogonal projections $\Phi(x, e)=$ $x \cdot e, e \in S^{d-1}$. For Euclidean distances, one can take $\Phi(x, y)=|x-y|$ and their result implies the following.

Theorem 1.1 (Peres, Schlag, (2000) ([15])).

$$
\begin{gathered}
\operatorname{dim}_{\mathcal{H}}\left(\left\{y \in \mathbb{R}^{d}: \operatorname{dim}_{\mathcal{H}}\left(\Delta^{y}(E)\right)<\beta\right\}\right) \leq d+\beta-\max \left\{\operatorname{dim}_{\mathcal{H}}(E), 1\right\}, \\
\operatorname{dim}_{\mathcal{H}}\left(\left\{y \in \mathbb{R}^{d}:\left|\Delta^{y}(E)\right|=0\right\}\right) \leq d+1-\operatorname{dim}_{\mathcal{H}}(E), \\
\operatorname{dim}_{\mathcal{H}}\left(\left\{y \in \mathbb{R}^{d}: \operatorname{Int}\left(\Delta^{y}(E)\right)=\emptyset\right\}\right) \leq d+2-\operatorname{dim}_{\mathcal{H}}(E) .
\end{gathered}
$$

In 2016, Iosevich, Taylor and Uriarte-Tuero ([7]) gave a straightforward proof of (1.3) for functions $\Phi(x, y)$ satisfying the Phong-Stein rotational curvature condition (1.1) in the range $\operatorname{dim}_{\mathcal{H}}(E)>\frac{d+1}{2}$. Shmerkin $([\mathbf{1 7}])$ recently proved that

$$
\operatorname{dim}_{\mathcal{H}}\left(\left\{y \in \mathbb{R}^{2}: \operatorname{dim}_{\mathcal{H}}\left(\Delta^{y}(E)\right)<1\right\}\right) \leq 1
$$

for any $E \subset \mathbb{R}^{2}$ such that $\operatorname{dim}_{\mathcal{H}}(E)=\operatorname{dim}_{P}(E)>1$, where $\operatorname{dim}_{P}(E)$ denotes the packing dimension of $E$.

It is interesting to note that the Peres-Schlag bound is in general sharp. One can simply take $\Phi(x, y)=x \cdot y$ and $E=A \times \mathbb{R}^{k} \times\{0\}^{d-k-1}$ where $A \subset \mathbb{R}$ has Hausdorff dimension $\beta$. Then $\operatorname{dim}_{\mathcal{H}}(E)=\beta+k$ while

$$
\left\{y \in \mathbb{R}^{d}: \operatorname{dim}_{\mathcal{H}}\left(\Delta^{y}(E)\right)<\beta\right\} \supset \mathbb{R} \times\{0\}^{k} \times \mathbb{R}^{d-k-1},
$$

whose Hausdorff dimension is $d-k=d+\beta-\operatorname{dim}_{\mathcal{H}}(E)$. Therefore, if we want to improve PeresSchlag's bound for, say Euclidean distances, we need a stronger assumption that rules out the case $\Phi(x, y)=x \cdot y$. 
Notice that the key difference between $|x-y|$ and $x \cdot y$ is that if $y$ is fixed, $\{x:|x-y|=1\}$ is a sphere with non-zero Gaussian curvature, while $\{x: x \cdot y=1\}$ is just a hyperplane. With this in mind, we obtain the following improvement of the aforementioned Peres-Schlag's result when $\operatorname{dim}_{\mathcal{H}}(E) \geq \frac{d+1}{2}$.

Definition 1.2. We say that $\Phi \in C^{\infty}\left(\mathbb{R}^{d} \times \mathbb{R}^{d}\right)$ satisfies Sogge's cinematic curvature condition ([18]) if

$$
\text { for any } t>0, x \in \mathbb{R}^{d},\left\{\nabla_{y} \Phi: \Phi(x, y)=t\right\} \text { has nonzero Gaussian curvature. }
$$

Theorem 1.3. Given $\Phi \in C^{\infty}\left(\mathbb{R}^{d} \times \mathbb{R}^{d}\right), E, F \subset \mathbb{R}^{d}$. Suppose $\Phi$ satisfies the Phong-Stein rotational curvature condition (1.1) and the cinematic curvature condition (1.5). Then there exists a probability measure $\mu_{F}$ on $F$ such that for $\mu_{F}$ a.e. $y \in F$,

$$
\begin{gathered}
\operatorname{dim}_{\mathcal{H}}\left(\Delta_{\Phi}^{y}(E)\right) \geq \beta \text { if } \operatorname{dim}_{\mathcal{H}}(E)+\frac{d-1}{d+1} \operatorname{dim}_{\mathcal{H}}(F)>d-1+\beta ; \\
\left|\Delta_{\Phi}^{y}(E)\right|>0 \text { if } \operatorname{dim}_{\mathcal{H}}(E)+\frac{d-1}{d+1} \operatorname{dim}_{\mathcal{H}}(F)>d ; \\
\operatorname{Int}\left(\Delta_{\Phi}^{y}(E)\right) \neq \emptyset \text { if } \operatorname{dim}_{\mathcal{H}}(E)+\frac{d-1}{d+1} \operatorname{dim}_{\mathcal{H}}(F)>d+1 .
\end{gathered}
$$

Improvement on pinned distance problem then follows easily.

Corollary 1.4. Given $\Phi \in C^{\infty}\left(\mathbb{R}^{d} \times \mathbb{R}^{d}\right), E \subset \mathbb{R}^{d}$. Suppose $\Omega \subset \mathbb{R}^{d}$ and $\Phi$ satisfies the Phong-Stein rotational curvature condition (1.1) and the cinematic curvature condition (1.5) on $E \times \Omega$. Then

$$
\begin{gathered}
\operatorname{dim}_{\mathcal{H}}\left(\left\{y \in \Omega: \operatorname{dim}_{\mathcal{H}}\left(\Delta_{\Phi}^{y}(E)\right)<\beta\right\}\right) \leq d+1+\frac{d+1}{d-1} \beta-\frac{d+1}{d-1} \operatorname{dim}_{\mathcal{H}}(E), \\
\operatorname{dim}_{\mathcal{H}}\left(\left\{y \in \Omega:\left|\Delta_{\Phi}^{y}(E)\right|=0\right\}\right) \leq \frac{d(d+1)}{d-1}-\frac{d+1}{d-1} \operatorname{dim}_{\mathcal{H}}(E), \\
\operatorname{dim}_{\mathcal{H}}\left(\left\{y \in \Omega: \operatorname{Int}\left(\Delta_{\Phi}^{y}(E)\right)=\emptyset\right\}\right) \leq \frac{(d+1)^{2}}{d-1}-\frac{d+1}{d-1} \operatorname{dim}_{\mathcal{H}}(E) .
\end{gathered}
$$

One can check that (1.9) improves (1.2) when $\operatorname{dim}_{\mathcal{H}}(E)>\frac{d-1}{2}+\beta ;(1.10)$ improves (1.3) when $\operatorname{dim}_{\mathcal{H}}(E)>\frac{d+1}{2}$ and (1.11) improves (1.4) when $\operatorname{dim}_{\mathcal{H}}(E)>\frac{d+3}{2}$.

In particular, when $\operatorname{dim}_{\mathcal{H}}(E)=d$, we have the following sharp corollary.

Corollary 1.5. Suppose $E \subset \mathbb{R}^{d}$, $\operatorname{dim}_{\mathcal{H}}(E)=d$. With notations above,

$$
\operatorname{dim}_{\mathcal{H}}\left(\left\{y \in \Omega:\left|\Delta_{\Phi}^{y}(E)\right|=0\right\}\right)=0 .
$$


1.2. Dimensions of slicing sets. Given a family of functions $\left\{\pi_{\lambda}\right\}_{\lambda \in \Lambda}$ and $E \subset \mathbb{R}^{d}$ such that $\pi_{\lambda}(x) \neq \pi_{\lambda^{\prime}}(x)$ whenever $\lambda \neq \lambda^{\prime}$, then $\left\{\pi_{\lambda}^{-1}(t)\right\}_{t \in \mathbb{R}}$ slices $E$ into pieces and we can study not only the size of images $\left\{\pi_{\lambda}(x): \lambda \in \Lambda, x \in E\right\}$, but also the size of those slices $E \cap\left\{\pi_{\lambda}^{-1}(t)\right\}$. The most classical result is due to Marstrand.

Theorem 1.6 (Marstrand, 1954). Suppose $E \subset \mathbb{R}^{2}$ is a Borel set and let $\pi_{\theta}(x)=x \cdot(\cos \theta, \sin \theta)$. Then for almost all $\theta \in[0,2 \pi)$,

$$
\begin{array}{r}
\operatorname{dim}_{\mathcal{H}}\left(\pi_{\theta}(E)\right)=\operatorname{dim}_{\mathcal{H}}(E) \text { if } \operatorname{dim}_{\mathcal{H}}(E) \leq 1 ; \\
\left|\pi_{\theta}(E)\right|>0 \text { if } \operatorname{dim}_{\mathcal{H}}(E)>1 .
\end{array}
$$

Moreover, when $\operatorname{dim}_{\mathcal{H}}(E)>1$, the typical lines with direction $\theta$ which intersect $E$ intersect it in dimension $\operatorname{dim}_{\mathcal{H}}(E)-1$, i.e.,

$$
\left|\left\{t \in \mathbb{R}: \operatorname{dim}_{\mathcal{H}}\left(\pi_{\theta}^{-1}(t) \cap E\right)=\operatorname{dim}_{\mathcal{H}}(E)-1\right\}\right|>0 \text {. }
$$

A variety of generalizations of this result have been obtained over the years. See, for example, [9] and the references contained therein.

What Peres and Schlag proved in [15] generalized (1.12) from orthogonal projections to a large class of functions so-called generalized projections, including, in particular, Euclidean distances $\pi_{\lambda}(x)=|x-\lambda|, x, \lambda \in \mathbb{R}^{d}$. In [13], Orponen generalized (1.13) from orthogonal projections to generalized projections.

Since Theorem 1.3 improves Peres-Schlag's result on dimensions of images of projections, it is natural to ask whether an improvement on dimensions of slicing sets can also be obtained. Its geometric meaning is that under the assumptions of Theorem 1.3, a typical distance $t$ occurs statistically often.

THEOREM 1.7. Under the assumptions of Theorem 1.3, with $E, F \subset \mathbb{R}^{d}$ and

$$
\operatorname{dim}_{\mathcal{H}}(E)+\frac{d+1}{d-1} \operatorname{dim}_{\mathcal{H}}(F)>d
$$

there exists a probability measure $\mu_{F}$ on $F$ such that for $\mu_{F}$-a.e. $y \in F$,

$$
|\{\Phi(x, y): x \in E\}|>0
$$

and

$$
\left|\left\{t: \operatorname{dim}_{\mathcal{H}}(\{x \in E: \Phi(x, y)=t\}) \geq \operatorname{dim}_{\mathcal{H}}(E)+\frac{d+1}{d-1} \operatorname{dim}_{\mathcal{H}}(F)-d\right\}\right|>0 .
$$

1.3. Local smoothing of Fourier integral operators. The study of Fourier integral operators arises in the study of the wave equation,

$$
\left\{\begin{array}{l}
\frac{\partial^{2} u}{\partial t^{2}}=\Delta u \\
\left.u\right|_{t=0}=f,\left.\frac{\partial u}{\partial t}\right|_{t=0}=0
\end{array}\right.
$$

whose solution is given by the real part of

$$
\mathcal{F} f(y, t):=\int e^{2 \pi i y \cdot \xi} e^{-2 \pi i t|\xi|} \hat{f}(\xi) d \xi .
$$

More generally, we may consider the Fourier integral operators (FIO), introduced by Hörmander in 1970s ([5]). In this paper, we only present a simple form of FIO for convenience. For general information, see e.g. [19] and the references therein. 
Definition 1.8. Suppose $\Phi \in C^{\infty}(U \times V)$, where $U, V \subset \mathbb{R}^{d}$ are open domains. For any $f \in C_{0}^{\infty}(V)$, one can define

$$
\begin{aligned}
\mathcal{F} f(y, t) & =\int_{\mathbb{R}^{d}}\left(\int_{\mathbb{R}} e^{-2 \pi i(\Phi(x, y)-t) \tau} d \tau\right) f(x) d x \\
& =\lim _{N \rightarrow \infty} \int_{\mathbb{R}^{d}}\left(\int_{-N}^{N} e^{-2 \pi i(\Phi(x, y)-t) \tau} d \tau\right) f(x) d x .
\end{aligned}
$$

Throughout this paper, we use the notation

$$
\alpha_{d, p, \gamma}=-\frac{d-1}{2}+\gamma+(d-1)\left|\frac{1}{2}-\frac{1}{p}\right| .
$$

It is known that if $\Phi$ satisfies the Phong-Stein rotational curvature condition (1.1), then for any fixed $t>0$,

$$
(I-\Delta)^{\frac{\gamma}{2}} \mathcal{F}(\cdot, t): L_{\alpha_{d, p, \gamma}}^{p}(U) \rightarrow L^{p}(V),
$$

for any $1<p<\infty$. This result is sharp if $t$ is fixed. But if $\Phi$ satisfies the cinematic curvature condition (1.5), there is a gain of regularity for $2<p<\infty$ by taking average in $t \approx 1$. This phenomenon is called local smoothing.

Theorem $1.9\left(L^{2} \rightarrow L^{p}\right.$ local smoothing estimate, Seeger, Sogge, Stein, 1991). Let $\mathcal{F}$ be as in Definition 1.8. Suppose $\Phi$ satisfies the Phong-Stein rotational curvature condition (1.1) and the cinematic curvature condition (1.5) on $U \times V$. Then

$$
\left\|\left(\int_{1}^{2}\left|(I-\Delta)^{\frac{\gamma}{2}} \mathcal{F} f(\cdot, t)\right|^{2} d t\right)^{\frac{1}{2}}\right\|_{L^{p}(V)} \lesssim\|f\|_{L_{\alpha_{d, p, \gamma}-\delta_{2}(d, p)}(U)},
$$

where

$$
\delta_{2}(d, p)= \begin{cases}\frac{1}{p}, & p \geq \frac{2(d+1)}{d-1} \\ \frac{1}{2}(d-1)\left(\frac{1}{2}-\frac{1}{p}\right), & 2<p \leq \frac{2(d+1)}{d-1} .\end{cases}
$$

It is known that $(1.14)$ does not hold with $\delta_{2}(d, p)=\frac{1}{p}$ for any $p<\frac{2(d+1)}{(d-1)}$. Also $L_{\alpha_{d, p, \gamma}-\frac{1}{p}-\epsilon}^{2}(U) \rightarrow$ $L^{p}\left(L^{2}([1,2])\right)(V)$ breaks down for any $\epsilon>0, p>2$.

It is more interesting to consider $L^{p} \rightarrow L^{p}$ local smoothing estimate because it is related to the Bochner-Riesz conjecture. Sogge's local smoothing conjecture $([\mathbf{1 8}])$ takes the following form in our setup.

Conjecture $1.10\left(L^{p} \rightarrow L^{p}\right.$ local smoothing estimate, Sogge, 1991). Let $\mathcal{F}$ be as in Definition 1.8. Suppose $\Phi$ satisfies the Phong-Stein rotational curvature condition (1.1) and the cinematic curvature condition (1.5) on $U \times V$. Then

$$
(I-\Delta)^{\frac{\gamma}{2}} \mathcal{F}: L_{\alpha_{d, p, \gamma}-\delta_{p}(d, p)+\epsilon}^{p}(U) \rightarrow L^{p}(V \times[1,2])
$$

for any $\epsilon>0$ with

$$
\delta_{p}(d, p)= \begin{cases}\frac{1}{p}, & p \geq \frac{2 d}{d-1} \\ (d-1)\left(\frac{1}{2}-\frac{1}{p}\right), & 2<p \leq \frac{2 d}{d-1}\end{cases}
$$


The best currently known result, accumulating efforts by Sogge ([18]), Mockenhaupt, Seeger, Sogge $([\mathbf{1 2}])$ and Wolff $([\mathbf{2 1}])$, is due to Bourgain and Demeter $([\mathbf{1}])$. They proved that (1.16) holds with

$$
\delta_{p}(d, p)= \begin{cases}\frac{1}{p}, & p \geq \frac{2(d+1)}{d-1} ; \\ \frac{1}{2}(d-1)\left(\frac{1}{2}-\frac{1}{p}\right), & 2<p \leq \frac{2(d+1)}{d-1} .\end{cases}
$$

It is known that $\delta_{p}(d, p)=\frac{1}{p}$ is the best possible for $p \geq \frac{2 d}{d-1}$ due to the sharpness of BochnerRiesz conjecture. There are also examples showing $\delta_{p}(d, p)>\frac{1}{p}$ fails for all $2<p<\infty$. In [11], Minicozzi and Sogge construct a metric $\Phi$ on odd dimensional spaces such that $\delta_{p}(d, p)=\frac{1}{p}$ works only if $p \geq \frac{2(d+2)}{d}$. Whether Sogge's conjecture is optimal in the range $p \geq \frac{2 d}{d-1}$ is an open question.

We will see that the proof of Theorem 1.3 relies on local smoothing estimates. Therefore, in turn, geometric counterexamples of Theorem 1.3 imply sharpness of local smoothing estimates.

Theorem 1.11 (Sharpness of Theorem 1.9). Suppose $\Phi$ satisfies assumptions in Theorem 1.9.

(i) For all $\Phi$,

$$
L_{\alpha_{d, p, \gamma}-\frac{1}{p}-\epsilon}^{2}(U) \rightarrow L^{p}\left(L^{2}([1,2])\right)(V)
$$

breaks down for any $\epsilon>0, p>2$.

(ii) In odd dimensions, there exists $\Phi$ to show that Theorem 1.9 is sharp for all $p>2$.

(iii) In even dimensions, there exists $\Phi$ such that (1.14) breaks down with any $\delta_{2}(d, p)>\frac{1}{2} d\left(\frac{1}{2}-\frac{1}{p}\right)$

THEOREM 1.12 (Sharpness of Conjecture 1.10). Suppose $\Phi$ satisfies assumptions in Conjecture 1.10

(i) For all $\Phi$,

$$
L_{\alpha_{d, p, \gamma}-\frac{1}{p}-\epsilon}^{p}(U) \rightarrow L^{p}(V \times[1,2])
$$

breaks down for any $\epsilon>0, p>2$.

(ii) In odd dimensions, there exists $\Phi$ to show that Conjecture 1.10 is the best possible for all $2<p \leq \frac{2 d}{d-1}$.

(iii) In even dimensions, there exists $\Phi$ such that (1.16) breaks down with any $\delta_{p}(d, p)>d\left(\frac{1}{2}-\frac{1}{p}\right)$.

\section{Notation.}

- $s_{E}$ denotes the Hausdorff dimension of $E \subset \mathbb{R}^{d}$.

- $X \lesssim Y$ means there exists a constant $C$ such that $X \leq C Y . X \lesssim_{\epsilon} Y$ means the implicit constant only depends on $\epsilon$.

- $\|\nu\|_{L_{\gamma}^{2}}^{2}:=\int|\hat{\nu}(\xi)|^{2}|\xi|^{2 \gamma} d \xi$.

- $\alpha_{d, p, \gamma}=-\frac{d-1}{2}+\gamma+(d-1)\left|\frac{1}{2}-\frac{1}{p}\right|$.

Acknowledgements. The second listed author would like to thank Professor Ka-Sing Lau for the financial support of research assistantship in Chinese University of Hong Kong.

\section{Proof of Theorem 1.3}

For any $E \subset \mathbb{R}^{n}$, there exists Frostman measures supported on it that reflects its Hausdorff dimension. 
Lemma 2.1 (Frostman Lemma, see e.g. [8]). Denote $\mathcal{H}^{s}$ as the s-dimensional Hausdorff measure and $E \subset \mathbb{R}^{n}$. Then $\mathcal{H}^{s}(E)>0$ if and only if there exists a probability measure $\mu$ on $E$, such that

$$
\mu(B(x, r)) \lesssim r^{s}, \forall x \in \mathbb{R}^{n}, \forall r>0
$$

The definition of Hausdorff dimension states that $\operatorname{dim}_{\mathcal{H}}(E)=\sup \left\{s: \mathcal{H}^{s}(E)>0\right\}$, denoted by $s_{E}$. So by Lemma 2.1, for any $E \subset \mathbb{R}^{d}$ and $\epsilon>0$, there exists $\mu_{E}$ on $E$ such that

$$
\mu_{E}(B(x, r)) \lesssim_{\epsilon} r^{s_{E}-\epsilon}, \forall x \in \mathbb{R}^{n}, \forall r>0
$$

To study the size of the support of a measure $\nu$, we need the following lemma.

Lemma 2.2 (see, e.g. Theorem 5.4 in $[\mathbf{1 0}]$ ). Suppose $\nu$ is a probability measure on $\mathbb{R}$ and $\|\nu\|_{L_{\gamma}^{2}}<\infty$.

(i) If $\gamma<0$, the support of $\nu$ has Hausdorff dimension at least $1+2 \gamma$.

(ii) If $\gamma \geq 0$, the support of $\nu$ has positive Lebesgue measure.

(iii) If $\gamma>\frac{1}{2}$, the support of $\nu$ has non-empty interior.

Fix $y \in \mathbb{R}^{d}$, define a measure $\nu_{y}$ on

$$
\Delta_{\Phi}^{y}(E)=\{\Phi(x, y): x \in E\}
$$

by

$$
\begin{aligned}
\int f(t) d \nu_{y}(t) & =\int f(\Phi(x, y)) d \mu_{E}(x) \\
& =\iint \epsilon^{2 \pi i \Phi(x, y) \tau} \hat{f}(\tau) d \tau d \mu_{E}(x) \\
& =\iint \epsilon^{2 \pi i \Phi(x, y) \tau}\left(\int e^{-2 \pi i t \tau} f(t) d t\right) d \tau d \mu_{E}(x) .
\end{aligned}
$$

Therefore, in the sense of distribution,

$$
d \nu_{y}(t)=\int\left(\int e^{-2 \pi i(\Phi(x, y)-t) \tau} d \tau\right) d \mu_{E}(x),
$$

which will be denoted as $\mathcal{F} \mu(y, t)$ as in Definition 1.8 .

By Lemma 2.2, to prove Theorem 1.3, it suffices to show that for any $F \subset \mathbb{R}^{d}$ of Hausdorff dimension $s_{F}>d+1+(1+2 \gamma) \frac{(d+1)}{d-1}-\frac{d+1}{d-1} s_{E}$ and for any Frostman measure $\mu_{F}$ on $F$ in (2.1),

$$
\int\left\|\nu_{y}\right\|_{L_{\gamma}^{2}} d \mu_{F}(y)=\int\left\|\partial_{t}^{\gamma} \mathcal{F} \mu(y, t)\right\|_{L^{2}(d t)} d \mu_{F}(y)<\infty .
$$

We first decompose $\mu_{E}, \mu_{F}$ into Littlewood-Paley pieces. There exists $\phi(\xi) \in C_{0}^{\infty}\left(\mathbb{R}^{d}\right)$, supported on $\left\{\frac{1}{2}<|\xi|<2\right\}$ such that

$$
\sum_{j=1}^{\infty} \phi\left(\frac{\xi}{2^{j}}\right)=1, \forall|\xi| \geq 1 .
$$


Denote $\phi_{j}(\cdot)=\phi\left(\frac{\dot{2}}{2^{j}}\right), \phi_{0}=1-\sum_{j=1}^{\infty} \phi_{j}, \widehat{\mu_{E}^{j}}=\widehat{\mu_{E}} \phi_{j}, \widehat{\mu_{F}^{j}}=\widehat{\mu_{F}} \phi_{j}$, then

$$
\mu_{E}=\sum_{j=0}^{\infty} \mu_{E}^{j}, \mu_{F}=\sum_{j=0}^{\infty} \mu_{F}^{j}
$$

and

$$
\begin{aligned}
\int\left\|\partial_{t}^{\gamma} \mathcal{F} \mu_{E}(y, t)\right\|_{L^{2}(d t)} d \mu_{F}(y) & \leq \sum_{j} \int\left\|\partial_{t}^{\gamma} \mathcal{F} \mu_{E}^{j}(y, t)\right\|_{L^{2}(d t)} d \mu_{F}(y) \\
& \leq \sum_{j}\left(\int\left\|\partial_{t}^{\gamma} \mathcal{F} \mu_{E}^{j}(y, t)\right\|_{L^{2}(d t)}^{2} d \mu_{F}(y)\right)^{\frac{1}{2}} \\
& =\sum_{j}\left(\sum_{j^{\prime}} \int\left\|\partial_{t}^{\gamma} \mathcal{F} \mu_{E}^{j}(y, t)\right\|_{L^{2}(d t)}^{2} \mu_{F}^{j^{\prime}}(y) d y\right)^{\frac{1}{2}}
\end{aligned}
$$

To complete the proof, we need the following two lemmas, whose proofs are given at the end of this section.

LEMma 2.3. There exists $j_{0}>0$ such that whenever $j^{\prime}>j+j_{0}$,

$$
\int\left\|\partial_{t}^{\gamma} \mathcal{F} \mu_{E}^{j}(y, t)\right\|_{L^{2}(d t)}^{2} \mu_{F}^{j^{\prime}}(y) d y \lesssim_{N} 2^{-j^{\prime} N}, \quad \forall N>0
$$

LEMMA 2.4.

$$
\left\|\mu_{E}^{j}\right\|_{L_{\alpha}^{p}} \lesssim \epsilon 2^{j\left(\alpha+\frac{d-s_{E}+\epsilon}{p^{\prime}}\right)}
$$

In particular,

$$
\int_{|\xi| \lesssim 2^{j}}\left|\widehat{\mu_{E}}(\xi)\right|^{2} d \xi \lesssim \epsilon 2^{j\left(d-s_{E}+\epsilon\right)}
$$

By Hölder's inequality, Theorem 1.9 and Lemma 2.4, for any $j, j^{\prime}>0$,

$$
\begin{aligned}
\int\left\|\partial_{t}^{\gamma} \mathcal{F} \mu_{E}^{j}(y, t)\right\|_{L^{2}(d t)}^{2} \mu_{F}^{j^{\prime}}(y) d y & \leq\left(\int\left\|\partial_{t}^{\gamma} \mathcal{F} \mu_{E}^{j}(y, t)\right\|_{L^{2}(d t)}^{2 q} d y\right)^{\frac{1}{2 q} \cdot 2}\left\|\mu_{F}^{j^{\prime}}\right\|_{L^{q^{\prime}}} \\
& \lesssim\left\|\mu_{E}^{j}\right\|_{L_{\alpha_{d, 2 q, \gamma}}^{2}-\delta_{2}(d, 2 q)}^{2}\left\|\mu_{F}^{j^{\prime}}\right\|_{L^{q^{\prime}}} \\
& \lesssim \epsilon 2^{2 j\left(-\frac{d-1}{2}+\gamma+(d-1)\left(\frac{1}{2}-\frac{1}{2 q}\right)-\delta_{2}(d, 2 q)+\frac{d-s_{E}+\epsilon}{2}\right)} \cdot 2^{j^{\prime} \frac{d-s_{F}+\epsilon}{q}} .
\end{aligned}
$$


Together with Lemma 2.3, (2.4) becomes

$$
\begin{aligned}
& \int\left\|\partial_{t}^{\gamma} \mathcal{F} \mu(y, t)\right\|_{L^{2}(d t)} d \mu_{F}(y) \\
\leq & \sum_{j}\left(\sum_{j^{\prime}} \int\left\|\partial_{t}^{\gamma} \mathcal{F} \mu_{E}^{j}(y, t)\right\|_{L^{2}(d t)}^{2} \mu_{F}^{j^{\prime}}(y) d y\right)^{\frac{1}{2}} \\
\leq & \sum_{j}\left(\sum_{j^{\prime}<j+j_{0}} \int\left\|\partial_{t}^{\gamma} \mathcal{F} \mu_{E}^{j}(y, t)\right\|_{L^{2}(d t)}^{2} \mu_{F}^{j^{\prime}}(y) d y+2^{-j^{\prime} N}\right)^{\frac{1}{2}} \\
\lesssim & \sum_{j}\left(\sum_{j^{\prime}<j+j_{0}} 2^{2 j\left(-\frac{d-1}{2}+\gamma+(d-1)\left(\frac{1}{2}-\frac{1}{2 q}\right)-\delta_{2}(d, 2 q)+\frac{d-s_{E}+\epsilon}{2}\right)} \cdot 2^{j^{\prime} \frac{d-s_{F}+\epsilon}{q}}+2^{-j^{\prime} N}\right)^{\frac{1}{2}} \\
\lesssim & \sum_{j} 2^{j\left(\gamma+\frac{1}{2 q}-\delta_{2}(d, 2 q)+\frac{d}{2}-\frac{s_{E}-\epsilon}{2}-\frac{s_{F}-\epsilon}{2 q}\right)} .
\end{aligned}
$$

Take $q=\frac{(d+1)}{d-1}$ and $\epsilon$ to be small enough, then $\delta_{2}(d, 2 q)=\frac{1}{2 q}$ and the integral above is finite whenever

as desired.

$$
s_{F}>d+1+(1+2 \gamma) \frac{(d+1)}{d-1}-\frac{d+1}{d-1} s_{E}
$$

PROOF OF Lemma 2.3. By interpolation we may assume $\gamma$ is an integer, then

$$
\partial_{t}^{\gamma} \mathcal{F} \mu_{E}^{j}(y, t)=\int_{\mathbb{R}^{d}}\left(\int_{\mathbb{R}} e^{-2 \pi i(\Phi(x, y)-t) \tau} \tau^{\gamma} d \tau\right) \mu_{E}^{j}(x) d x
$$

and by Plancherel in $t$,

$$
\begin{aligned}
& \int\left\|\partial_{t}^{\gamma} \mathcal{F} \mu_{E}^{j}(y, t)\right\|_{L^{2}(d t)}^{2} \mu_{F}^{j^{\prime}}(y) d y \\
= & \iint\left(\int_{\mathbb{R}}\left(\int_{\mathbb{R}^{d}} e^{-2 \pi i \Phi(x, y) \tau} \tau^{\gamma} \mu_{E}^{j}(x) d x\right) e^{2 \pi i t \tau} d \tau\right)^{2} d t \mu_{F}^{j^{\prime}}(y) d y \\
= & \iint\left(\int_{\mathbb{R}^{d}} e^{-2 \pi i \Phi(x, y) \tau} \tau^{\gamma} \mu_{E}^{j}(x) d x\right)^{2} d \tau \mu_{F}^{j^{\prime}}(y) d y \\
= & \iiint \int e^{-2 \pi i\left(\Phi(x, y)-\Phi\left(x^{\prime}, y\right)\right) \tau} \tau^{2 \gamma} \mu_{E}^{j}(x) \mu_{E}^{j}\left(x^{\prime}\right) \mu_{F}^{j^{\prime}}(y) d x d x^{\prime} d y d \tau .
\end{aligned}
$$

By the definition of Littlewood-Paley decomposition,

$$
\left|\mu^{j}(x)\right|=2^{j d} \mid \int \hat{\phi}\left(2^{j}(x-z) d \mu(z) \mid \leq C_{N} 2^{j d} \int\left(1+2^{j}|x-z|\right)^{-N} d \mu(z) .\right.
$$

So it suffices to consider $x, x^{\prime}, y$ in (2.7) in a bounded domain. So it approximately equals

$$
\int\left(\int_{|x|,\left|x^{\prime}\right|,|y| \lesssim 1} e^{-2 \pi i\left(\Phi(x, y) \tau-\Phi\left(x^{\prime}, y\right) \tau-x \cdot \xi-x^{\prime} \cdot \xi^{\prime}-y \cdot \eta\right)} d x d x^{\prime} d y\right) \widehat{\mu_{E}^{j}}(\xi) \widehat{\mu_{E}^{j}}\left(\xi^{\prime}\right) \widehat{\mu_{F}^{j^{\prime}}}(\eta) d \xi d \xi^{\prime} d \eta \tau^{2 \gamma} d \tau \text {. }
$$


Denote the phase function as $\varphi$, i.e.,

$$
\varphi=\Phi(x, y) \tau-\Phi\left(x^{\prime}, y\right) \tau-x \cdot \xi-x^{\prime} \cdot \xi^{\prime}-y \cdot \eta .
$$

Then

$$
\begin{aligned}
\nabla_{x} \varphi & =\tau \nabla_{x} \Phi(x, y)-\xi \\
\nabla_{x^{\prime}} \varphi & =-\tau \nabla_{x^{\prime}} \Phi\left(x^{\prime}, y\right)-\xi^{\prime} \\
\nabla_{y} \varphi & =\tau\left(\nabla_{y} \Phi(x, y)-\nabla_{y} \Phi\left(x^{\prime}, y\right)\right)-\eta .
\end{aligned}
$$

where $|\xi| \approx\left|\xi^{\prime}\right| \approx 2^{j},|\eta| \approx 2^{j^{\prime}}$.

Since the Phong-Stein rotational curvature condition (1.1) holds, $\left|\nabla_{x} \Phi\right| \approx\left|\nabla_{x^{\prime}} \Phi\right| \approx\left|\nabla_{y} \Phi\right| \approx 1$.

If $\left|\nabla_{y} \varphi\right| \gtrsim 2^{j^{\prime}}$, the lemma follows by integration by parts. If not, $\tau$ must be $\gtrsim 2^{j^{\prime}}$. Therefore if $j^{\prime}>j+j_{0}$, it follows that $\left|\nabla_{x} \varphi\right| \approx\left|\nabla_{x^{\prime}} \varphi\right| \gtrsim 2^{j^{\prime}}$ and the Lemma follows by integration by parts.

PRoOf OF Lemma 2.4. Again by interpolation we may assume $\alpha$ is an even integer. Denote $\phi_{j}(\cdot)=\phi\left(\frac{\dot{2}}{2^{j}}\right)$. Then $\mu^{j}=\widehat{\phi_{j}} * \mu$ and

$$
\Delta^{\frac{\alpha}{2}} \mu_{E}^{j}(x)=\left(\Delta^{\frac{\alpha}{2}} \widehat{\phi_{j}}\right) * \mu(x) .
$$

It is easy to see

$$
\left\|\Delta^{\frac{\alpha}{2}} \widehat{\phi_{j}}\right\|_{L^{1}} \lesssim 2^{\alpha j}
$$

Therefore

$$
\left\|\mu_{E}^{j}\right\|_{L_{\alpha}^{1}}=\left\|\left(\Delta^{\frac{\alpha}{2}} \widehat{\phi_{j}}\right) * \mu\right\|_{L^{1}} \leq\left\|\Delta^{\frac{\alpha}{2}} \widehat{\phi_{j}}\right\|_{L^{1}}\|\mu\|_{L^{1}} \lesssim 2^{j \alpha} .
$$

For $L_{\alpha}^{\infty}$ norm, notice

$$
\left|\left(\Delta^{\frac{\alpha}{2}} \widehat{\phi_{j}}\right) * \mu(x)\right|=2^{j \alpha} 2^{j d}\left|\int\left(\Delta^{\frac{\alpha}{2}} \widehat{\phi}\right)\left(2^{j}(x-z)\right) d \mu(z)\right| \lesssim_{\epsilon} 2^{j\left(d-s_{E}+\alpha+\epsilon\right)}
$$

where the last inequality follows by $(2.1)$ and the fact $\left|\left(\Delta^{\frac{\alpha}{2}} \widehat{\phi}\right)\left(2^{j}(x-z)\right)\right| \lesssim_{N}\left(1+2^{j}|x-z|\right)^{-N}$. This upper bound is uniformly in $x$ so we have

$$
\left\|\mu_{E}^{j}\right\|_{L_{\alpha}^{\infty} \lesssim \epsilon} 2^{j\left(d-s_{E}+\alpha+\epsilon\right)}
$$

and the lemma follows by interpolation.

\section{Proof of Theorem 1.7}

In the last section we proved that $\nu_{y}(t)$ on

$$
\Delta_{\Phi}^{y}(E)=\{\Phi(x, y): x \in E\}
$$

defined in (2.2), is in fact an $L^{2}$ function for $\mu_{F}$-a.e. $y \in F$. With this in mind one can define the slicing measure $\mu_{E}^{y, t}$ on $\{x \in E: \Phi(x, y)=t\}$ as

$$
\int f d \mu_{E}^{y, t}=\iint e^{-2 \pi i(\Phi(x, y)-t) \tau} d \tau f(x) d \mu_{E}(x) .
$$

Notice $\mu_{E}^{y, t}$ is a well-defined measure for $\mu_{F} \times \mathcal{L}^{1}$-a.e. $(y, t) \in F \times \mathbb{R}$ because by definition

$$
\int d \mu_{E}^{y, t}=\nu_{y}(t)<\infty
$$


for $\mu_{F} \times \mathcal{L}^{1}$-a.e. $(y, t) \in F \times \mathbb{R}$. Also because

$$
\int \nu_{y}(t) d t=\int d \mu_{E}(x)=1,
$$

$\left\{t \in \mathbb{R}: \int \mu_{E}^{y, t}>0\right\}$ has positive Lebesgue measure for $\mu_{F}$-a.e. $y \in F$.

Define the $\sigma$-energy of $\mu_{E}^{y, t}$ as

$$
I_{\sigma}\left(\mu_{E}^{y, t}\right)=\iint|u-v|^{-\sigma} d \mu_{E}^{y, t}(u) d \mu_{E}^{y, t}(v)=\left.c_{\sigma, d} \int \widehat{\mid \mu_{E}^{y, t}}(\xi)\right|^{2}|\xi|^{-d+\sigma} d \xi
$$

It is known that the finiteness of $I_{\sigma}\left(\mu_{E}^{y, t}\right)$ implies the Hausdorff dimension of the support of $\mu_{E}^{y, t}$ is at least $\sigma$ (see, e.g. [8]). Thus to prove Theorem 1.7, it suffices to show

$$
\iint I_{\sigma}\left(\mu_{E}^{y, t}\right) d t d \mu_{F}(y)<\infty
$$

whenever $\sigma<s_{E}+\frac{d+1}{d-1} s_{F}-d$.

By the definition of $I_{\sigma}\left(\mu_{E}^{y, t}\right)$,

$$
\begin{aligned}
& \iint I_{\sigma}\left(\mu_{E}^{y, t}\right) d t d \mu_{F}(y) \\
= & \iiint\left|\widehat{\mu_{E}^{y, t}}(\xi)\right|^{2}|\xi|^{-d+\sigma} d \xi d t d \mu_{F}(y) \\
= & \iiint\left|\iint e^{-2 \pi i(\Phi(x, y)-t) \tau} d \tau e^{-2 \pi i x \cdot \xi} d \mu_{E}(x)\right|^{2}|\xi|^{-d+\sigma} d \xi d t d \mu_{F}(y) .
\end{aligned}
$$

Denote $\mu_{E}^{\xi}=e^{-2 \pi i x \cdot \xi} \mu_{E}$. By Plancherel in $t,(3.2)$ becomes

$$
\begin{aligned}
& \iiint\left|\int e^{-2 \pi i \Phi(x, y) \tau} d \mu_{E}^{\xi}(x)\right|^{2}|\xi|^{-d+\sigma} d \xi d \tau d \mu_{F}(y) \\
= & \int \cdots \int e^{-2 \pi i\left(\left(\Phi(x, y)-\Phi\left(x^{\prime}, y\right)\right) \tau\right.} d \mu_{E}^{\xi}(x) d \mu_{E}^{\xi}\left(x^{\prime}\right)|\xi|^{-d+\sigma} d \xi d \tau d \mu_{F}(y) \\
= & \int\left(\int e^{-2 \pi i\left(\left(\Phi(x, y)-\Phi\left(x^{\prime}, y\right)\right) \tau-x \cdot \eta-x^{\prime} \cdot \eta^{\prime}-y \cdot \zeta\right)} d x d x^{\prime} d y\right) \widehat{\mu_{E}^{\xi}}(\eta) \widehat{\mu_{E}^{\xi}}\left(\eta^{\prime}\right) \widehat{\mu_{F}}(\zeta)|\xi|^{-d+\sigma} d \xi d \eta d \eta^{\prime} d \zeta d \tau .
\end{aligned}
$$

Since $E, F$ are both compact sets, $x, x^{\prime}, y$ above lie in a bounded domain. Denote $\varphi$ as the phase function in the integral above, i.e.,

$$
\varphi=\left(\Phi(x, y)-\Phi\left(x^{\prime}, y\right)\right) \tau-x \cdot \eta-x^{\prime} \cdot \eta^{\prime}-y \cdot \zeta .
$$

Then

$$
\begin{aligned}
\nabla_{x} \varphi & =\tau \nabla_{x} \Phi(x, y)-\eta, \\
\nabla_{x^{\prime}} \varphi & =-\tau \nabla_{x^{\prime}} \Phi\left(x^{\prime}, y\right)-\eta^{\prime}, \\
\nabla_{y} \varphi & =\tau\left(\nabla_{y} \Phi(x, y)-\nabla_{y} \Phi\left(x^{\prime}, y\right)\right)-\zeta .
\end{aligned}
$$

Since the Phong-Stein rotational curvature condition (1.1) holds, $\left|\nabla_{x} \Phi\right| \approx\left|\nabla_{x^{\prime}} \Phi\right| \approx\left|\nabla_{y} \Phi\right| \approx 1$.

When $|\nabla \varphi|=0, \tau \approx|\eta| \approx\left|\eta^{\prime}\right| \gtrsim|\zeta|$. Thus it suffices to consider the domains where $|\eta| \approx\left|\eta^{\prime}\right| \approx$ $2^{j_{1}},|\zeta| \approx 2^{j_{2}}, j_{1} \geq j_{2}$. 
For each fixed $\xi$, denote

$$
\mu_{E}^{\xi, j_{1}}=\left(\widehat{\mu_{E}^{\xi}}(\cdot) \phi\left(\frac{\cdot}{2^{j_{1}}}\right)\right)^{\vee}=\left(\widehat{\mu_{E}}(\cdot+\xi) \phi\left(\frac{\cdot}{2^{j_{1}}}\right)\right)^{\vee} .
$$

The discussion above implies that, to show the finiteness of (3.2), it suffices to consider

$$
\begin{aligned}
& \sum_{j_{1} \geq j_{2}} \iint\left(\int\left|\iint e^{-2 \pi i(\Phi(x, y)-t) \tau} d \tau \mu_{E}^{\xi, j_{1}}(x) d x\right|^{2} d t\right) \mu_{F}^{j_{2}}(y) d y|\xi|^{-d+\sigma} d \xi \\
= & \sum_{j_{1} \geq j_{2}} \int\left(\int\left(\int\left|\mathcal{F} \mu_{E}^{\xi, j_{1}}(y, t)\right|^{2} d t\right) \mu_{F}^{j_{2}}(y) d y\right)|\xi|^{-d+\sigma} d \xi .
\end{aligned}
$$

Fix $\xi$, apply Hölder's inequality in $d y$ with $q>1$. It is less than or equal to

$$
\sum_{j_{1} \geq j_{2}}\left\|\mu_{F}^{j_{2}}\right\|_{L^{q^{\prime}}} \int\left(\int\left(\int\left|\mathcal{F} \mu_{E}^{\xi, j_{1}}(y, t)\right|^{2} d t\right)^{\frac{1}{2} \cdot 2 q} d y\right)^{\frac{1}{2 q} \cdot 2}|\xi|^{-d+\sigma} d \xi
$$

Then by Lemma 2.4 and the $L^{2} \rightarrow L^{p}$ sharp local smoothing estimate, with $2 q=\frac{2(d+1)}{d-1}$, this integral is bounded above by

$$
\begin{aligned}
& \sum_{j_{1} \geq j_{2}} 2^{j_{2} \frac{d-s_{F}+\epsilon}{q}} \int\left\|\mu_{E}^{\xi, j_{1}}(y, t)\right\|_{L_{\alpha_{d, 2 q, 0}-\frac{1}{2 q}}^{2}}^{2}|\xi|^{-d+\sigma} d \xi \\
= & \sum_{j_{1}} 2^{j_{1} \frac{d-s_{F}+\epsilon}{q}} \int\left\|\mu_{E}^{\xi, j_{1}}(y, t)\right\|_{L_{\alpha_{d, 2 q, 0}-\frac{1}{2 q}}^{2}}^{2}|\xi|^{-d+\sigma} d \xi .
\end{aligned}
$$

So to prove (3.1), it suffices to show (3.4) is finite.

By the definition of $\mu_{E}^{\xi, j_{1}}$,

$$
\widehat{\mu_{E}^{\xi, j_{1}}}(\eta)=\widehat{\mu_{E}}(\eta+\xi) \phi\left(\frac{\eta}{2^{j_{1}}}\right)
$$

So if we take $2 q=\frac{2(d+1)}{d-1}$,

$$
\begin{aligned}
\int \|\left.\mu_{E}^{\xi, j_{1}}(y, t)\right|_{L_{\alpha_{d, 2 q}-\frac{1}{2 q}}^{2}} ^{2}|\xi|^{-d+\sigma} d \xi & =\iint_{|\eta| \approx 2^{j_{1}}}\left|\widehat{\mu_{E}^{\xi}}(\eta)\right|^{2}|\eta|^{-\frac{d(d-1)}{d+1}} d \eta|\xi|^{-d+\sigma} d \xi \\
& \approx 2^{-j_{1} \frac{d(d-1)}{d+1}} \iint_{|\eta| \approx 2^{j_{1}}}\left|\widehat{\mu_{E}}(\eta+\xi)\right|^{2} d \eta|\xi|^{-d+\sigma} d \xi \\
& =2^{-j_{1} \frac{d(d-1)}{d+1}}\left(\int_{|\xi| \lesssim 2^{j_{1}}} \int_{|\eta| \approx 2^{j_{1}}}+\sum_{j_{3} \geq j_{1}} \int_{|\xi| \approx 2^{j_{3}}} \int_{|\eta| \approx 2^{j_{1}}}\right) \\
& =2^{-j_{1} \frac{d(d-1)}{d+1}}(I+I I) .
\end{aligned}
$$


For $I$, since $|\xi| \lesssim 2^{j_{1}},|\eta| \approx 2^{j_{1}}$, we have $|\eta+\xi| \lesssim 2^{j_{1}} \approx|\eta|$. Then by changing variables $\eta^{\prime}=\eta+\xi$ and Lemma 2.4,

$$
\begin{aligned}
I & =\int_{|\xi| \lesssim 2^{j_{1}}} \int_{|\eta| \approx 2^{j_{1}}}\left|\widehat{\mu_{E}}(\eta+\xi)\right|^{2} d \eta|\xi|^{-d+\sigma} d \xi \\
& \lesssim \int_{|\xi| \lesssim 2^{j_{1}}} \int_{\left|\eta^{\prime}\right| \lesssim 2^{j_{1}}}\left|\widehat{\mu_{E}}\left(\eta^{\prime}\right)\right|^{2} d \eta^{\prime}|\xi|^{-d+\sigma} d \xi \\
& \lesssim \epsilon 2^{j_{1}\left(d-s_{E}+\epsilon+\sigma\right)} .
\end{aligned}
$$

For $I I$, since $|\xi| \approx 2^{j_{3}},|\eta| \approx 2^{j_{1}}, j_{3} \geq j_{1}$, it follows $|\eta+\xi| \lesssim 2^{j_{3}} \approx|\xi|$. Then by changing variables $\xi^{\prime}=\eta+\xi$ and Lemma 2.4,

$$
\begin{aligned}
I I & =\int_{|\xi| \approx 2^{j_{3}}} \int_{|\eta| \approx 2^{j_{1}}}\left|\widehat{\widehat{\mu_{E}}}(\eta+\xi)\right|^{2} d \eta|\xi|^{-d+\sigma} d \xi \\
& \lesssim 2^{j_{3}(-d+\sigma)} \int_{|\eta| \approx 2^{j_{1}}}\left(\int_{|\xi| \approx 2^{j_{3}}}\left|\widehat{\mu_{E}}(\eta+\xi)\right|^{2} d \xi\right) d \eta \\
& \lesssim 2^{j_{3}(-d+\sigma)} \int_{|\eta| \approx 2^{j_{1}}}\left(\int_{\left|\xi^{\prime}\right| \approx 2^{j_{3}}}\left|\widehat{\mu_{E}}\left(\xi^{\prime}\right)\right|^{2} d \xi^{\prime}\right) d \eta \\
& \lesssim \epsilon 2^{j_{3}(-d+\sigma)} 2^{j_{3}\left(d-s_{E}+\epsilon\right)} 2^{j_{1} d} \\
& =2^{j_{3}\left(\sigma-s_{E}+\epsilon\right)} 2^{j_{1} d} .
\end{aligned}
$$

These estimates of $I, I I$ give us an upper bound of (3.5). Thus (3.4) is bounded above, with $2 q=\frac{2(d+1)}{d-1}$, by

$$
\sum_{j_{1}} 2^{j_{1}\left(d-s_{F}+\epsilon\right) \frac{(d-1)}{d+1}} 2^{-j_{1} \frac{d(d-1)}{d+1}}\left(2^{j_{1}\left(d-s_{E}+\epsilon+\sigma\right)}+\sum_{j_{1} \leq j_{3}} 2^{j_{3}\left(\sigma-s_{E}+\epsilon\right)} 2^{j_{1} d}\right) .
$$

Since $\sigma$ is the expected dimension of slicing measures of $E$, we may assume $\sigma<s_{E}$, then the sum is no bigger than

$$
\sum_{j_{1}} 2^{j_{1}\left(d-s_{F}+\epsilon\right) \frac{(d-1)}{d+1}} 2^{-j_{1} \frac{d(d-1)}{d+1}} 2^{j_{1}\left(d-s_{E}+\epsilon+\sigma\right)},
$$

which is finite whenever $\sigma<s_{E}+\frac{d-1}{d+1} s_{F}-d$ and $\epsilon$ is sufficiently small.

\section{Proof of Theorem 1.11 and Theorem 1.12}

We prove Theorem 1.11 first. If we run the proof of Theorem 1.3, without taking a specific value of $q$ in the last step, it follows that

$$
\operatorname{dim}_{\mathcal{H}}\left(\left\{y \in V:\left|\Delta_{\Phi}^{y}(E)\right|=0\right\}\right) \leq q d+1-2 q \delta_{2}(d, 2 q)-q \operatorname{dim}_{\mathcal{H}}(E), \forall q>1 .
$$
means

For $(i)$, if $\delta_{2}(d, 2 q)>\frac{1}{2 q}$, the right hand side of (4.1) is negative when $\operatorname{dim}_{\mathcal{H}}(E)=d$, which

$$
\left\{y \in V:\left|\Delta_{\Phi}^{y}(E)\right|=0\right\}=\emptyset .
$$


However, one can take $E=\left\{x: \Phi\left(x, y_{0}\right) \in A\right\}$, where $y_{0} \in V$ is fixed and $A \subset \mathbb{R}$ has Hausdorff dimension 1 but Lebesgue measure 0 . Then $\operatorname{dim}_{\mathcal{H}}(E)=d$, while $\left|\Delta_{\Phi}^{y_{0}}(E)\right|=|A|=0$. Contradiction.

For $(i i)$, say $d=2 k+1$, one can take $\Phi(x, y)$ to be the $(k+1, k)$ - Minkowski distance between $x$ and $y$, i.e.

$$
\Phi(x, y)=\|x-y\|_{k+1, k}=\sum_{i=1}^{d}(-1)^{i+1}\left(x_{i}-y_{i}\right)^{2}
$$

and

$$
E=A \times\left\{(t, t) \in \mathbb{R}^{2}: t \in \mathbb{R}\right\}^{k},
$$

where $A \subset \mathbb{R}$ has Hausdorff dimension 1 but Lebesgue measure 0 . Then for any point

$$
y \in\left\{\left(y_{1}, \ldots, y_{2 k+1}\right) \in \mathbb{R}^{2 k+1}: y_{2 j}=y_{2 j+1}, 1 \leq j \leq k\right\},
$$

whose Hausdorff dimension is $\frac{d+1}{2}$, it is easy to see that $\left|\Delta_{\Phi}^{y}(E)\right|=\left|y_{1}\right| \cdot|A|=0$. This means

$$
\operatorname{dim}_{\mathcal{H}}\left(\left\{y \in \Omega:\left|\Delta_{\Phi}^{y}(E)\right|=0\right\}\right) \geq \frac{d+1}{2} .
$$

Compared with $(4.1), \delta_{2}(d, 2 q)$ has to be less than or equal to $\frac{1}{2}(d-1)\left(\frac{1}{2}-\frac{1}{2 q}\right)$.

For $(i i i)$, say $d=2 k$, one can take

$$
\Phi(x, y)=x_{1} y_{1}+\sum_{i=2}^{d}(-1)^{i}\left(x_{i}-y_{i}\right)^{2}
$$

and

$$
E=A \times[1,2] \times\left\{(t, t) \in \mathbb{R}^{2}: t \in \mathbb{R}\right\}^{k-1},
$$

where $A \subset \mathbb{R}$ has Hausdorff dimension 1 but Lebesgue measure 0 . Then for any

$$
y \in\left\{\left(y_{1}, \ldots, y_{2 k}\right) \in \mathbb{R}^{2 k}: y_{2 j-1}=j_{2 j}, 2 \leq j \leq k\right\},
$$

whose Hausdorff dimension is $\frac{d}{2}$, one can check that $\left|\Delta_{\Phi}^{y}(E)\right|=0$. This means

$$
\operatorname{dim}_{\mathcal{H}}\left(\left\{y \in \Omega:\left|\Delta_{\Phi}^{y}(E)\right|=0\right\}\right) \geq \frac{d}{2} .
$$

Compare with $(4.1), \delta_{2}(d, 2 q)$ has to be less than or equal to $(d-1)\left(\frac{1}{2}-\frac{1}{2 q}\right)$.

For Theorem 1.12, we apply Conjecture 1.10 instead of Theorem 1.9 in the proof of Theorem 1.3. More precisely, we apply Hölder's inequality

$$
\begin{aligned}
\left|\int\left\|\partial_{t}^{\gamma} \mathcal{F} \mu_{E}^{j}(y, t)\right\|_{L^{2}(d t)}^{2} \mu_{F}^{j^{\prime}}(y) d y\right| & \leq \int\left\|\partial_{t}^{\gamma} \mathcal{F} \mu_{E}^{j}(y, t)\right\|_{L^{p}(d t)}^{2}\left|\mu_{F}^{j^{\prime}}(y)\right| d y \\
& \leq\left\|\partial_{t}^{\gamma} \mathcal{F} \mu_{E}^{j}(y, t)\right\|_{L^{p}(V \times[1,2])}^{2}\left\|\mu_{F}^{j^{\prime}}\right\|_{L^{(p / 2)^{\prime}}(V)} \\
& \lesssim\left\|\mu_{E}^{j}\right\|_{L_{\alpha_{d, p, \gamma}-\delta_{p}(d, p)}^{2}}\left\|\mu_{F}^{j^{\prime}}\right\|_{L^{(p / 2)^{\prime}}} \\
& \lesssim_{\epsilon} 2^{2 j\left(-\frac{d-1}{2}+\gamma+(d-1)\left(\frac{1}{2}-\frac{1}{p}\right)-\delta_{p}(d, p)+\frac{d-s_{E}+\epsilon}{p^{\prime}}\right)} \cdot 2^{j^{\prime} \frac{2\left(d-s_{F}+\epsilon\right)}{p}} .
\end{aligned}
$$

It follows that, for any $p>2$,

$$
\operatorname{dim}_{\mathcal{H}}\left(\left\{y \in V:\left|\Delta_{\Phi}^{y}(E)\right|=0\right\}\right) \leq \frac{p}{p^{\prime}} d+1-p \delta_{p}(d, p)-\frac{p}{p^{\prime}} \operatorname{dim}_{\mathcal{H}}(E) .
$$


Then Theorem 1.12 follows with the same counterexamples in the proof of Theorem 1.11.

\section{References}

[1] J. Bourgain and C. Demeter. The proof of the $l^{2}$ decoupling conjecture. Ann. of Math. (2), 182(1):351-389, 2015. 6

[2] M. B. Erdogan. A bilinear Fourier extension theorem and applications to the distance set problem. Int. Math. Res. Not., (23):1411-1425, 2005. 1

[3] S. Eswarathasan, A. Iosevich, and K. Taylor. Fourier integral operators, fractal sets, and the regular value theorem. Adv. Math., 228(4):2385-2402, 2011. 2

[4] K. J. Falconer. On the Hausdorff dimensions of distance sets. Mathematika, 32(2):206-212, 1985. 1

[5] L. Hörmander. Fourier integral operators. I. Acta Math., 127(1-2):79-183, 1971. 4

[6] A. Iosevich and B. Liu. Falconer distance problem, additive energy and cartesian products. Ann. Acad. Sci. Fenn. Math., 41(2):579-585, 2016. 1

[7] A. Iosevich, K. Taylor, and I. Uriarte-Tuero. Pinned geometric configurations in euclidean space and riemannian manifolds. https://arxiv.org/pdf/1610.00349v1.pdf, 2016. 2

[8] P. Mattila. Geometry of Sets and Measures in Euclidean Spaces: Fractals and Rectifiability, volume 44 of Cambridge Studies in Advanced Mathematics. Cambridge University Press, Cambridge, 1995. 7, 11

[9] P. Mattila. Recent progress on dimensions of projections. In Geometry and analysis of fractals, volume 88 of Springer Proc. Math. Stat., pages 283-301. Springer, Heidelberg, 2014. 4

[10] P. Mattila. Fourier analysis and Hausdorff dimension, volume 150. Cambridge University Press, 2015. 7

[11] W. P. Minicozzi, II and C. D. Sogge. Negative results for Nikodym maximal functions and related oscillatory integrals in curved space. Math. Res. Lett., 4(2-3):221-237, 1997. 6

[12] G. Mockenhaupt, A. Seeger, and C. D. Sogge. Local smoothing of Fourier integral operators and Carleson-Sjölin estimates. J. Amer. Math. Soc., 6(1):65-130, 1993. 6

[13] T. Orponen. Slicing sets and measures, and the dimension of exceptional parameters. J. Geom. Anal., 24(1):4780, 2014. 4

[14] T. Orponen. On the distance sets of Ahlfors-David regular sets. Adv. Math., 307:1029-1045, 2017. 1

[15] Y. Peres and W. Schlag. Smoothness of projections, Bernoulli convolutions, and the dimension of exceptions. Duke Math. J., 102(2):193-251, 2000. 2, 4

[16] P. Shmerkin. On distance sets, box-counting and ahlfors-regular sets. arXiv:1604.00308, 2016. 1

[17] P. Shmerkin. On the hausdorff dimension of pinned distance sets. arXiv preprint arXiv:1706.00131, 2017. 2

[18] C. D. Sogge. Propagation of singularities and maximal functions in the plane. Invent. Math., 104(2):349-376, 1991. 3, 5, 6

[19] C. D. Sogge. Fourier integrals in classical analysis, volume 105 of Cambridge Tracts in Mathematics. Cambridge University Press, Cambridge, 1993. 4

[20] T. Wolff. Decay of circular means of Fourier transforms of measures. Internat. Math. Res. Notices, (10):547-567, 1999. 1

[21] T. Wolff. Local smoothing type estimates on $L^{p}$ for large p. Geom. Funct. Anal., 10(5):1237-1288, 2000. 6

E-mail address: iosevich@math.rochester.edu

Department of Mathematics, University of Rochester, Rochester, NY

E-mail address: bochen.liu@rochester.edu

Department of Mathematics, University of Rochester, Rochester, Ny 